\title{
Convergence Analysis of EFOP Estimate Based on Frequency Domain Smoothing
}

\author{
Yulai Zhang, Kueiming Lo, and Yang Su \\ School of Software, Tsinghua University, Tsinghua National Laboratory for Information Science and Technology, Beijing 100084, China \\ Correspondence should be addressed to Yulai Zhang; zhangyl08@mails.tsinghua.edu.cn
}

Received 8 March 2013; Revised 21 April 2013; Accepted 24 April 2013

Academic Editor: Graziano Chesi

Copyright ( 2013 Yulai Zhang et al. This is an open access article distributed under the Creative Commons Attribution License, which permits unrestricted use, distribution, and reproduction in any medium, provided the original work is properly cited.

\begin{abstract}
The classic methods for frequency domain transfer function estimation such as the Empirical Transfer Function Estimate (ETFE) and cross spectral method do not work well when the noise signal is complex. Combines the time domain and frequency domain methods the Empirical Frequency-domain Optimal Parameter (EFOP) Estimate was presented. It could improve the precision of system's transfer function estimation and identification efficiency. The convergence of the EFOP based on frequency domain smoothing is investigated in this paper. The transfer function is weighted by a frequency window and the GPE criterion is extended to the integral form. Convergence rate and consistent properties for the EFOP estimate are given. Finally, some simulation results are included to illustrate the advantage of the EFOP based smoothing method.
\end{abstract}

\section{Introduction}

Parametric and nonparametric estimations in system identification or signal processing are both of great importance. Most of these algorithms use input and output data to give a modeling of the target system. The modeling of linear system transfer function is a non-parameter method, and it is one of the most important basic problems in control theory. From the beginning of modern system identification and signal processing, many nonparametric methods in frequency domain have been proposed, such as ETFE [1], power spectrum estimation [2], and so on. Frequency domain window functions $[3,4]$ are used in power spectrum estimation. In the past few decades, algorithms for window function selection are developed, such as adaptive smoothing method in [5] and least square estimation for frequency function in [6]. Discussion of the relationship between time domain methods and frequency domain methods can be found in [7]. EFOP [8, 9] is an algorithm combines the time domain and frequency domain. A new criterion named General Prediction Error (GPE) is proposed and implemented in EFOP. This algorithm obtains the global optimal transfer function in frequency domain by minimizing the GPE criterion. The advantage of this kind of algorithm includes a faster rate of convergence as well as better identification qualities. The algorithms given in $[8,9]$ are parametric in time domain, so they cannot be directly used in the identification of transfer function in frequency domain. Since frequency domain methods have their own advantages such as robustness for outliers in data [10], it is necessary to develop frequency domain methods.

In this article we analysis the convergence of transfer function estimation based on EFOP and frequency domain window function smoothing technic. Firstly, the identification of transfer function is derived with GPE criterion in EFOP; secondly, the spectrum analysis is used to calculated the predict error and a window function to smoothing the transfer function in frequency domain. The consistence and convergence properties of this procedure are analyzed in this paper. It is proofed that this new smoothing method is asymptotically unbiased and the rate of convergence is fast. Simulations in the end show that the method proposed in this paper gives better results than the ETFE on identification accuracy under large noises.

For the rest of this article, preliminaries including EFOP and GPE are introduced in Section 2; the frequency domain smoothing procedure is derived in Section 3; its rate of convergence and estimator's bias properties are analyzed in Section 4; simulation results are given in Section 5 and Section 6 is the conclusions. 
The contributions of this article include the following.

(i) Extending the GPE criterion in EFOP to its integral form.

(ii) Development of EFOP smoothing method for transfer function estimation.

(iii) The rate of convergence for this new method is proved.

\section{Preliminaries}

Most input and output signals for system identification procedures are sampled from application systems in industry or laboratory. Denote $x(t)_{1}^{N}$ as a data sequence, where $N$ is the total number of samples that is, the length of the data sequence. The Discrete Fourier Transformation (DFT) of $x(t)_{1}^{N}$ is

$$
\begin{array}{r}
X_{N}(k)=\operatorname{DFT}\{x(t)\}_{1}^{N}=\frac{1}{\sqrt{N}} \sum_{t=1}^{N} x(t) \omega^{-t k}, \\
k=1,2,3, \ldots, N,
\end{array}
$$

where $\omega=\exp (2 i \pi / N)$. The Inverse Discrete Fourier Transformation (IDFT) can also be denoted as:

$$
\begin{aligned}
x(k) & =\operatorname{IDFT}\left\{X_{N}(k)\right\}_{1}^{N} \\
& =\frac{1}{\sqrt{N}} \sum_{t=1}^{N} X_{N}(k) \omega^{t k}, \quad t=1,2,3, \ldots, N
\end{aligned}
$$

Provided that $\{x(t)\}_{1}^{N}$ and $\{z(t)\}_{1-N}^{N}$ are signal sequence with same length, the convolution of then can be written as

$$
(x * z)(t)=\frac{1}{\sqrt{N}} \sum_{l=1}^{N} x(t) z(l-t), \quad t=1,2,3, \ldots, N
$$

Lemma 1 (Parse Val equation). Provided that $X_{N}(k)=$ $\operatorname{DFT}\{x(t)\}_{1}^{N}=1 / \sqrt{N} \sum_{t=1}^{N} x(t) \omega^{-t k}, k=1,2,3, \ldots, N$, that is, $x(t)$ and $X(k)$ are DFT pairs. One can obtain

$$
\sum_{t=1}^{N}|x(t)|^{2}=\sum_{k=1}^{N}\left|X_{N}(k)\right|^{2}
$$

Lemma 2. For periodic signal $\{z(t)\}_{1-N}^{N}, z(t)=z(t+N), 1-$ $N \leq t \leq 0$, we can obtain:

$$
\operatorname{DFT}\{(x * z)(t)\}_{1}^{N}=X_{N}(k) Z_{N}(k),
$$

where $X_{N}(k)=\operatorname{DFT}\{x(t)\}_{1}^{N}, Z_{N}(k)=\operatorname{DFT}\{z(t)\}_{1}^{N}$.
Lemma 3. Provided that $D=\left\{\left(a_{1}, a_{2}, \ldots, a_{N}\right) \mid \sum_{k=1}^{N} a_{k}=\right.$ $\left.M, a_{k} \geq 0\right\}, M$ is a constant, $\left\{\xi_{k}\right\}_{1}^{N}$ is stochastic i.d.d white noise and $E \xi_{k}^{2}=\lambda_{k}$. Let $\xi=\sum_{t=1}^{N} a_{k} \xi_{k}$, E $\xi^{2}$ reaches its minimum value when the coefficient satisfies $a_{k}=M\left(\lambda_{k} \sum_{k=1}^{N}\left(1 / \lambda_{k}\right)\right)^{-1}$.

This lemma can be simply obtained by using Lagrange method.

Denote $u(t)$ as an input data sequence, and $y(t)$ as an output one. A black box linear model can be expressed as

$$
A(q) y(t)=B(q) u(t)+\frac{C(q)}{D(q)} e_{0}(t)
$$

where $A(q)=1+\sum_{k=1}^{n_{a}} a_{k} q^{-k}, B(q)=\sum_{k=1}^{n_{b}} b_{k} q^{-k}, C(q)=$ $1+\sum_{k=1}^{n_{c}} c_{k} q^{-k}$, and $D(q)=1+\sum_{k=1}^{n_{d}} d_{k} q^{-k}$.

Rewrite this equation as

$$
y(t)=G(q, \theta) u(t)+H(q, \theta) e_{0}(t),
$$

where $G(q, \theta)=B(q) / A(q), H(q, \theta)=C(q) / A(q) D(q)$.

Denote $y(t \mid \theta)$ as the predictor of output $y(t)$ :

$$
y(t \mid \theta)=H^{-1}(q, \theta) G(q, \theta) u(t)+\left(1-H^{-1}(q, \theta)\right) y(t)
$$

$\theta$ is the parameter of the black box model here, and $\varepsilon(t, \theta)=$ $y(t)-y(t \mid \theta)=H^{-1}(q, \theta)(y(t)-G(q, \theta) u(t))$ is the predict error, which can also be written as

$$
\varepsilon(t, \theta)=\frac{A(q) D(q)}{C(q)} y(t)-\frac{B(q) D(q)}{C(q)} u(t) .
$$

The predict error vector can be presented as

$$
\beta(N, \theta)=(\varepsilon(1, \theta), \varepsilon(2, \theta), \ldots, \varepsilon(N, \theta))^{T} .
$$

Define a criterion function

$$
J=\frac{1}{N} \sum_{k=1}^{N} L(\varepsilon(t, \theta), \theta, t) .
$$

The estimation of the model under this criterion can be presented as

$$
\widehat{\theta}_{N}=\arg \min _{\theta} J(\beta(t, \theta) ; N ; \theta) .
$$

First we denote that the transfer function obtained by ETFE is $\widehat{G}_{\mathrm{NETFE}}\left(\omega^{k}\right)$, and the error of this estimation is calculated as

$$
\delta_{N}(k, \theta)=\widehat{G}_{\text {NETFE }}\left(\omega^{k}\right)-G_{N}\left(\omega^{k}\right), \quad k=1,2,3, \ldots, N .
$$

Lemma 4. Provided that the variance of $e_{0}(t)$ in (7) is $\lambda$ and it is a white noise, thus one obtains

$$
\begin{gathered}
E \delta_{N}(k, \theta)=0, \quad k=1,2,3, \ldots, N, \\
E \delta_{N}(k, \theta) \overline{\delta_{N}(l, \theta)} \\
\quad= \begin{cases}\lambda\left|\frac{C \omega^{k}}{A \omega^{k} D \omega^{k} U_{N}(k)}\right|^{2} & \text { for } k=l, \\
0 & \text { for } k \neq l,\end{cases}
\end{gathered}
$$

where $U_{N}(k)=\operatorname{DFT}\{u(t)\}_{1}^{N}$. 
Corollary 5. Under the assumption of Lemma 4, one can obtain the following equation:

$$
\begin{gathered}
\arg \min _{\Omega} E\left|\sum_{t=1}^{N} g_{k} \delta_{N}(k, \theta)\right|^{2} \\
=\arg \min _{\Omega} E \sum_{t=1}^{N} g_{k}^{2}\left|\delta_{N}(k, \theta)\right|^{2} \\
=\left(g_{1}^{*}, g_{2}^{*}, \ldots g_{N}^{*}\right),
\end{gathered}
$$

where

$$
g_{k}^{*}=\left|\frac{A\left(\omega^{k}\right) D\left(\omega^{k}\right) U_{N}(k)}{C\left(\omega^{k}\right)}\right|^{2}
$$

This can be derived from Lemma 3. We substitute the constant $M$ in Lemma 3 by

$$
M=\sum_{l=1}^{N}\left|\frac{A\left(\omega^{l}\right) D\left(\omega^{l}\right) U_{N}(l)}{C\left(\omega^{l}\right)}\right|^{2}
$$

in an off-line procedure, so $M$ can be taken as a constant.

Theorem 6. Consider

$$
\begin{aligned}
& \underset{\Omega}{\arg \min } E\left|\sum_{t=1}^{N} g_{k} \delta_{N}(k, \theta)\right|^{2} \\
& =\beta^{T}(N, \theta) Q(N, \theta) \beta(N, \theta),
\end{aligned}
$$

where $Q(N, \theta)=(1 / N) \sum_{s=1}^{N} \psi(s, \theta) \psi^{T}(s, \theta)$ which is the weight matrix, and

$$
\begin{gathered}
\psi(s, \theta)=\left(v_{e}(s-1, \theta), v_{e}(s-2, \theta), \ldots, v_{e}(s-N, \theta)\right)^{T}, \\
s=1,2,3, \ldots, N, \\
v_{e}(t, \theta)= \begin{cases}v(t, \theta), & \text { if } 1 \leq t \leq N \\
v(t+N, \theta), & \text { if } 1-N \leq t \leq 0 .\end{cases}
\end{gathered}
$$

Signal $\{v(t, \theta)\}_{1}^{N}$ is introduced by

$$
C(q) v(t, \theta)=A(q) D(q) u(t) .
$$

The proof can be completed by using Corollary 5 and Lemma 1 in this section, and it is similar to the proof in [11].

More details of EFOP and GPE can be can be found in [11]. EFOP gets the estimator by minimizing
$\beta^{T}(N, \theta) Q(N, \theta) \beta(N, \theta)$, which is the GPE criterion [8]

$$
\widehat{\theta}=\left(\Phi^{T}(N) Q(N) \Phi(N)\right)^{-1} \Phi^{T}(N) Q(N) Y(N),
$$

where $Q(N)=(1 / N) \sum_{t=1}^{N} \bar{\psi}(t, \theta) \bar{\psi}^{T}(t, \theta)$ and $\Phi(N)$ is composed of regression vectors

$$
\Phi(N)=(\phi(1), \phi(2), \ldots, \phi(N))^{T}
$$

and $\phi(k)=\left(-y(k-1),-y(k-2), \ldots,-y\left(k-n_{a}\right), u(k-1), u(k-\right.$ 2), ..., u(k-n $\left.n_{b}\right)$.

\section{New Method Based on EFOP and Frequency Domain Smoothing}

3.1. Representing Criterion in Frequency Domain. Rewrite $V_{N}\left(\theta, Z^{N}\right)$ in frequency domain as

$$
\begin{aligned}
V_{N}\left(\theta, Z^{N}\right)= & \arg \min E\left|\sum_{t=1}^{N} g_{k} \delta_{N}(k, \theta)\right|^{2} \\
= & \beta^{T}(N, \theta) Q(N, \theta) \beta(N, \theta) \\
= & \sum_{s=1}^{N}\left|\frac{U_{N}(s)}{H\left(\omega^{s}\right)}\right|^{4}\left|\delta_{N}(s, \theta)\right|^{2} \\
= & \sum_{s=1}^{N}\left|\frac{U_{N}(s)}{H\left(\omega^{s}\right)}\right|^{4}\left|\widehat{G}_{N}\left(\omega^{s}\right)-G_{N}\left(\omega^{s}\right)\right|^{2} \\
= & \sum_{s=1}^{N}\left|\widehat{G}_{\mathrm{NETFE}}\left(e^{2 \pi i k / N}\right)-G_{N}\left(e^{2 \pi i k / N}, \theta\right)\right|^{2} \\
& \quad \times Q_{N}\left(\frac{2 \pi i k}{N}, \theta\right),
\end{aligned}
$$

where $Q_{N}(\omega, \theta)=\left|U_{N}(\omega) / H\left(e^{i \omega}, \theta\right)\right|^{4}$. Since the frequency domain transfer function is actually a continuous function, the integrity form can be presented as

$$
\begin{aligned}
& V_{N}\left(\theta, Z^{N}\right) \\
& \quad \approx \frac{1}{2 \pi} \int_{-\pi}^{\pi}\left|\widehat{G}_{\text {NETFE }}\left(e^{i \omega}, \theta\right)-G_{N}\left(e^{i \omega}, \theta\right)\right|^{2} Q_{N}(\omega, \theta) d \omega .
\end{aligned}
$$

3.2. Algorithm with Frequency Domain Window. Denote $W_{\gamma}(\omega)$ as the window function. Window function can be seen as a way to perform weighting in frequency domain. If the width of the window function is relatively large, the variance of $G_{N}\left(e^{i \omega}, \theta\right)$ will be small. But a wider frequency window will make the bias of $G_{N}\left(e^{i \omega}, \theta\right)$ larger. So there is a tradeoff between the variance and bias of the estimated transfer function. A scalar $\gamma$ is introduced to adjust this 
tradeoff. A smaller $\gamma$ represents a wider window function. Some of the $W_{\gamma}(\omega)$ s properties are written as follows:

$$
\begin{array}{cl}
\int_{-\pi}^{\pi} W_{\gamma}(\xi) d \xi=1 ; & \int_{-\pi}^{\pi} \xi W_{\gamma}(\xi) d \xi=0 ; \\
\int_{-\pi}^{\pi} \xi^{2} W_{\gamma}(\xi) d \xi=M(\gamma) ; & \int_{-\pi}^{\pi} W_{\gamma}^{2}(\xi) d \xi=\frac{1}{2 \pi} \bar{W}(\gamma) .
\end{array}
$$

Theorem 7. If $\hat{\theta}_{N}=\arg \min _{\theta, \eta} \int_{-\pi}^{\pi}\left(\mid \widehat{G}_{\text {NETFE }}\left(e^{i \omega}, \theta\right)-\right.$ $\left.\left.G\left(e^{i \omega}, \theta\right)\right|^{2}\left|U_{N}(\omega)\right|^{4} /\left|H\left(e^{i \omega}, \eta\right)\right|^{4}\right) d \omega$, the systems transfer function can be estimated as

$$
\begin{aligned}
& G_{\text {NEFOP }}\left(e^{i \omega_{k}}\right) \\
& =\frac{\int_{-\pi}^{\pi} W_{\gamma}\left(\omega-\omega_{k}\right)\left|U_{N}(\omega)\right|^{4} \widehat{G}_{N E T F E}\left(e^{i \omega}, \theta\right) d \omega}{\int_{-\pi}^{\pi} W_{\gamma}\left(\omega-\omega_{k}\right)\left|U_{N}(\omega)\right|^{4} d \omega},
\end{aligned}
$$

where $W_{\gamma}(\omega)$ is the frequency domain window function and $G_{\text {NETFE }}\left(e^{i \omega}, \theta\right)$ is the estimated ETFE transfer function.

Proof. Rewrite $G\left(e^{i \omega}, \theta\right)$ as

$$
\begin{gathered}
G\left(e^{i \omega}, \theta\right)=\sum_{k=1}^{N}\left(g_{k}^{R}+i g_{k}^{I}\right) W_{\gamma}\left(\omega-\omega_{k}\right), \\
\theta_{k}=\left[g_{1}^{R}, g_{1}^{I}, \ldots g_{n}^{R}, g_{n}^{I}\right]^{T}
\end{gathered}
$$

$g_{k}^{R}$ and $g_{k}^{I}$ are the real and imaginary parts of $\theta_{k}$, respectively.

$$
\begin{aligned}
& \frac{\partial}{\partial \theta_{k}} \int_{-\pi}^{\pi} \frac{\left|\widehat{G}_{\text {NETFE }}\left(e^{i \omega}, \theta\right)-G\left(e^{i \omega}, \theta\right)\right|^{2}\left|U_{N}(\omega)\right|^{4}}{\left|H\left(e^{i \omega}, \eta\right)\right|^{4}} d \omega \\
& =\frac{\partial}{\partial \theta_{k}} \int_{-\pi}^{\pi} \frac{\left|\widehat{G}_{\text {NETFE }}\left(e^{i \omega}, \theta\right)-\sum_{k=1}^{N}\left(g_{k}^{R}+i g_{k}^{I}\right) W_{\gamma}\left(\omega-\omega_{k}\right)\right|^{2}\left|U_{N}(\omega)\right|^{4}}{\left|H\left(e^{i \omega}, \eta\right)\right|^{4}} d \omega \\
& =-2 \int_{-\pi}^{\pi} \frac{\left(\widehat{G}_{\text {NETFE }}\left(e^{i \omega}, \theta\right)-G\left(e^{i \omega}, \theta\right)\right) W_{\gamma}\left(\omega-\omega_{k}\right)\left|U_{N}(\omega)\right|^{4}}{\left|H\left(e^{i \omega}, \eta\right)\right|^{4}} d \omega=0 .
\end{aligned}
$$

Since $G\left(e^{i \omega}, \eta\right) W_{\gamma}\left(\omega-\omega_{k}\right) \approx G\left(e^{i \omega_{k}}, \eta\right) W_{\gamma}\left(\omega-\omega_{k}\right)$, so we have

$$
\begin{aligned}
& G_{\text {NEFOP }}\left(e^{i \omega_{k}}\right) \\
& \quad=\frac{\int_{-\pi}^{\pi} W_{\gamma}\left(\omega-\omega_{k}\right)\left|U_{N}(\omega)\right|^{4} \widehat{G}_{\text {NETFE }}\left(e^{i \omega}, \theta\right) d \omega}{\int_{-\pi}^{\pi} W_{\gamma}\left(\omega-\omega_{k}\right)\left|U_{N}(\omega)\right|^{4} d \omega} .
\end{aligned}
$$

The new algorithm is obtained by Theorem 7 . Figure 1 is the explanation of algorithm procedure. In Figure $1, G_{N}$ and $G_{D}$ are the numerator and denominator of the final result, respectively.

Elementary operations such as add and division are not explicitly shown in the chart.

\section{Consistency and Convergence Analysis}

The theoretical analysis of consistency and convergence for the proposed method are investigated in this section. It is proved that the estimation result is asymptotically unbiased and will converge to the true system transfer function.
4.1. Consistency and Bias. From the expression of $G\left(e^{i \omega_{k}}\right)$, we can obtain:

$$
\begin{aligned}
E \widehat{G}_{\text {NEFOP }}\left(e^{i \omega_{0}}\right) & \\
= & \frac{\int_{-\pi}^{\pi} W_{\gamma}\left(\xi-\omega_{0}\right)\left|\Phi_{u}(\xi)\right|^{2}}{\int_{-\pi}^{\pi} W_{\gamma}\left(\xi-\omega_{0}\right)\left|\Phi_{u}(\xi)\right|^{2} d \xi} \\
& \times \frac{\left[G_{0}\left(e^{i \xi}\right)+\rho_{1}(N) / U_{N}(\xi)\right] d \xi}{\int_{-\pi}^{\pi} W_{\gamma}\left(\xi-\omega_{0}\right)\left|\Phi_{u}(\xi)\right|^{2} d \xi} \\
\approx & \frac{\int_{-\pi}^{\pi} W_{\gamma}\left(\xi-\omega_{0}\right)\left|\Phi_{u}(\xi)\right|^{2} G_{0}\left(e^{i \xi}\right) d \xi}{\int_{-\pi}^{\pi} W_{\gamma}\left(\xi-\omega_{0}\right)\left|\Phi_{u}(\xi)\right|^{2} d \xi}
\end{aligned}
$$

where $\rho_{1}(N)$ is defined as

$$
E \widehat{G}_{\mathrm{NEFOP}}\left(e^{i \omega}\right)=G_{0}\left(e^{i \omega}\right)+\frac{\rho_{1}(N)}{U_{N}(\omega)}
$$




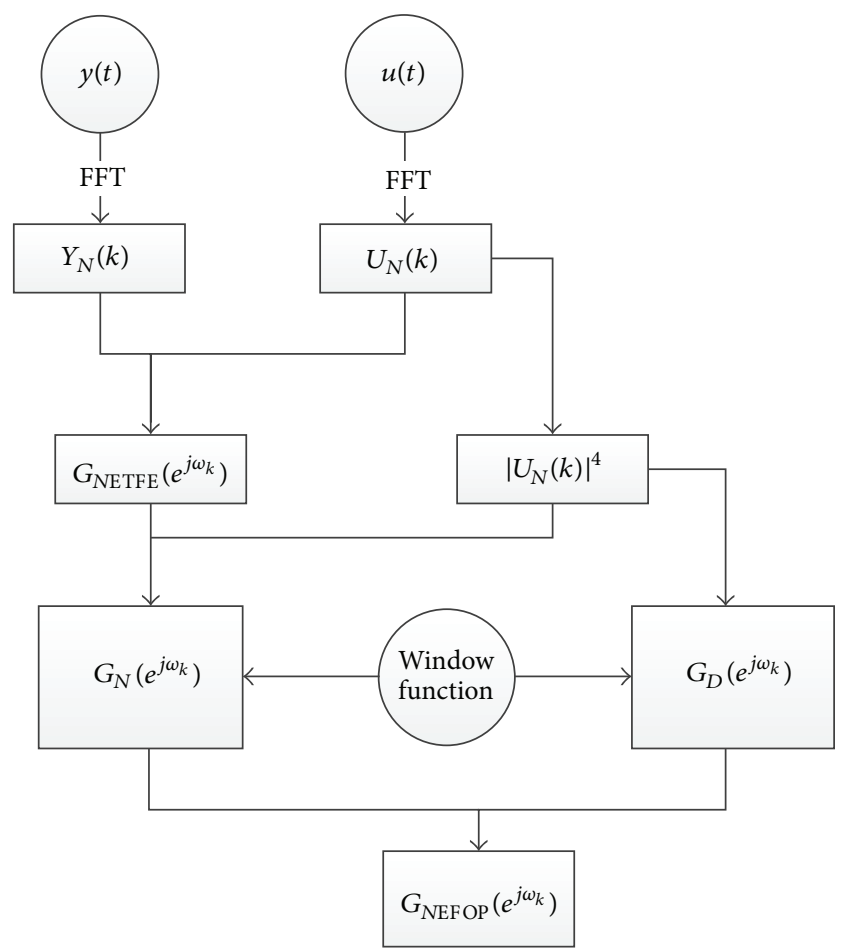

FIgURE 1: Algorithm procedure.

$\left|\rho_{1}(N)\right| \leq C_{1} / \sqrt{N}$ and $C_{1}=\left(2 \sum_{k=1}^{\infty}\left|k g_{0}(k)\right|\right) \cdot \max |u(t)|$. Given the Taylor expansion:

$$
\begin{aligned}
G_{0}\left(e^{i \xi}\right) \approx & G_{0}\left(e^{i \omega_{0}}\right)+\left(\xi-\omega_{0}\right) G_{0}^{\prime}\left(e^{i \omega_{0}}\right) \\
& +\frac{1}{2}\left(\xi-\omega_{0}\right)^{2} G_{0}^{\prime \prime}\left(e^{i \omega_{0}}\right), \\
\Phi_{0}^{2}(\xi) \approx & \Phi_{u}^{2}\left(\omega_{0}\right)+2\left(\xi-\omega_{0}\right) \Phi_{u}\left(\omega_{0}\right) \Phi_{u}^{\prime}\left(\omega_{0}\right) \\
+ & \left(\xi-\omega_{0}\right)^{2}\left[\Phi_{u}^{\prime}\left(\omega_{0}\right) \Phi_{u}^{\prime}\left(\omega_{0}\right)\right. \\
& \left.+\Phi_{u}\left(\omega_{0}\right) \Phi_{u}^{\prime \prime}\left(\omega_{0}\right)\right] .
\end{aligned}
$$

Note that the window function $W_{\gamma}$ should satisfy the following equations:

$$
\begin{array}{cl}
\int_{-\pi}^{\pi} W_{\gamma}(\xi) d \xi=1 ; & \int_{-\pi}^{\pi} \xi W_{\gamma}(\xi) d \xi=0 ; \\
\int_{-\pi}^{\pi} \xi^{2} W_{\gamma}(\xi) d \xi=M(\gamma) ; & \int_{-\pi}^{\pi}|\xi|^{3} W_{\gamma}(\xi) d \xi=C_{3}(\gamma) ;
\end{array}
$$

We can obtain the numerator of $E \widehat{G}_{N}\left(e^{i \omega_{0}}\right)$ as:

$$
\begin{aligned}
G_{0}\left(e^{i \omega_{0}}\right) \Phi_{u}^{2}\left(\omega_{0}\right) & \\
+M(\gamma)[ & G_{0}\left(e^{i \omega_{0}}\right) \Phi_{u}^{\prime}\left(\omega_{0}\right) \Phi_{u}^{\prime}\left(\omega_{0}\right) \\
& +G_{0}\left(e^{i \omega_{0}}\right) \Phi_{u}\left(\omega_{0}\right) \Phi_{u}^{\prime \prime}\left(\omega_{0}\right) \\
& +2 G_{0}^{\prime}\left(e^{i \omega_{0}}\right) \Phi_{u}\left(\omega_{0}\right) \Phi_{u}^{\prime}\left(\omega_{0}\right) \\
& \left.+\frac{1}{2} \Phi_{u}^{2}\left(\omega_{0}\right) G_{0}^{\prime \prime}\left(e^{i \omega_{0}}\right)\right] .
\end{aligned}
$$

And the denominator of $E \widehat{G}_{N}\left(e^{i \omega_{0}}\right)$ as:

$$
\Phi_{u}^{2}\left(\omega_{0}\right)+2 M(\gamma)\left[\Phi_{u}^{\prime}\left(\omega_{0}\right) \Phi_{u}^{\prime}\left(\omega_{0}\right)+\Phi_{u}\left(\omega_{0}\right) \Phi_{u}^{\prime \prime}\left(\omega_{0}\right)\right] .
$$

For sake of convenience, we denote $G_{0}^{\prime}\left(e^{i \omega_{0}}\right) \Phi_{u}\left(\omega_{0}\right) \Phi_{u}^{\prime}\left(\omega_{0}\right)+$ $(1 / 2) \Phi_{u}^{2}\left(\omega_{0}\right) G_{0}^{\prime \prime}\left(e^{i \omega_{0}}\right)-G_{0}\left(e^{i \omega_{0}}\right) \Phi_{u}\left(\omega_{0}\right) \Phi_{u}^{\prime \prime}\left(\omega_{0}\right)$ as $\Omega$ and $\Phi_{u}^{\prime}\left(\omega_{0}\right) \Phi_{u}^{\prime}\left(\omega_{0}\right)+\Phi_{u}\left(\omega_{0}\right) \Phi_{u}^{\prime \prime}\left(\omega_{0}\right)$ as $\Upsilon$. So the equation can be rewritten as:

$$
E \widehat{G}_{\mathrm{NEFOP}}\left(e^{i \omega_{0}}\right)=\frac{G_{0}\left(e^{i \omega_{0}}\right) \Phi_{u}^{2}\left(\omega_{0}\right)+M(\gamma) \Omega}{\Phi_{u}^{2}\left(\omega_{0}\right)+M(\gamma) \Upsilon}
$$

From the above equation, we can easily get that when $\gamma \rightarrow$ $\infty$ and $M(\gamma) \rightarrow 0$ :

$$
E \widehat{G}_{\mathrm{NEFOP}}\left(e^{i \omega_{0}}\right) \approx G_{0}\left(e^{i \omega_{0}}\right)
$$

Based on the property of the window function, we can obtain the following inequality:

$$
\begin{aligned}
& E \widehat{G}_{\mathrm{NEFOP}}\left(e^{i \omega_{0}}\right)-G_{0}\left(e^{i \omega_{0}}\right) \\
& <M(\gamma)\left[2 G_{0}^{\prime}\left(e^{i \omega_{0}}\right) \frac{\Phi_{u}^{\prime}\left(\omega_{0}\right)}{\Phi_{u}\left(\omega_{0}\right)}+\frac{1}{2} G_{0}^{\prime \prime}\left(e^{i \omega_{0}}\right)\right] \\
& +\underset{\gamma \rightarrow \infty}{O\left(C_{3}(\gamma)\right)}+\underset{N \rightarrow \infty}{\left(\frac{1}{\sqrt{N}}\right) .}
\end{aligned}
$$

When the first-order and second-order derivatives of the transfer function are bounded, the estimator $\widehat{G}_{\mathrm{NEFOP}}\left(e^{i \omega_{0}}\right)$ is asymptotically unbiased.

4.2. Variance Analysis and Rate of Convergence. From the results of previous sections, we have:

$$
\begin{aligned}
\widehat{G}_{\text {NEFOP }}\left(e^{i \omega_{0}}\right)-E \widehat{G}_{\text {NEFOP }}\left(e^{i \omega_{0}}\right) \\
\approx \frac{\int_{-\pi}^{\pi} W_{\gamma}\left(\xi-\omega_{0}\right)\left|U_{N}(\xi)\right|^{4}\left(V_{N}(\xi) / U_{N}(\xi)\right) d \xi}{\int_{-\pi}^{\pi} W_{\gamma}\left(\xi-\omega_{0}\right)\left|U_{N}(\xi)\right|^{4} d \xi} .
\end{aligned}
$$


The nominator of (39) can be written as

$$
\begin{aligned}
\int_{-\pi}^{\pi} W_{\gamma}\left(\xi-\omega_{0}\right)\left|U_{N}(\xi)\right|^{2} \overline{U_{N}}(\xi) V_{N}(\xi) d \xi \\
\approx \frac{2 \pi}{N} \sum_{k=-(N / 2)+1}^{N / 2} W_{\gamma}\left(\frac{2 \pi k}{N}-\omega_{0}\right) \\
\times\left|U_{N}\left(\frac{2 \pi k}{N}\right)\right|^{2} \overline{U_{N}}\left(\frac{2 \pi k}{N}\right) \\
\times V_{N}\left(\frac{2 \pi k}{N}\right) \triangleq A_{N},
\end{aligned}
$$

$$
\begin{aligned}
E A_{N} \overline{A_{N}} & =\frac{4 \pi^{2}}{N^{2}} \sum_{k} \sum_{l} W_{\gamma}\left(\frac{2 \pi k}{N}-\omega_{0}\right)\left|U_{N}\left(\frac{2 \pi k}{N}\right)\right|^{2} \\
& \times\left|U_{N}\left(-\frac{2 \pi l}{N}\right)\right|^{2} \overline{U_{N}}\left(\frac{2 \pi k}{N}\right) \\
= & \frac{4 \pi^{2}}{N^{2}}\left[W_{\gamma}\left(\frac{2 \pi k}{N}-\omega_{0}\right)\right]^{2}\left|U_{N}\left(\frac{2 \pi l}{N}\right) E V_{N}\left(\frac{2 \pi k}{N}\right)\right|_{N}\left(-\frac{2 \pi l}{N}\right) \\
& \times\left[\Phi_{v}\left(\frac{2 \pi k}{N}\right)+\rho_{2}(N)\right] \\
\approx & \frac{4 \pi^{2}}{N^{2}}\left[W_{\gamma}\left(\frac{2 \pi k}{N}-\omega_{0}\right)\right]^{2}\left|U_{N}\left(\frac{2 \pi k}{N}\right)\right|^{4} \Phi_{v}\left(\frac{2 \pi k}{N}\right),
\end{aligned}
$$

where $\rho_{2}(N) \leq 2 C / N$. Rewrite it in the integral form

$$
\begin{aligned}
E A_{N} \overline{A_{N}} & \approx \frac{2 \pi}{N} \int_{-\pi}^{\pi} W_{\gamma}^{2}\left(\xi-\omega_{0}\right) \Phi_{u}^{2}(\xi) \Phi_{v}(\xi) d \xi \\
& \approx \frac{1}{N} \bar{W}(\gamma) \Phi_{u}^{2}\left(\omega_{0}\right) \Phi_{v}\left(\omega_{0}\right) .
\end{aligned}
$$

And the denominator part can be written approximately as $\Phi_{u}^{2}\left(\omega_{0}\right)$. Notice $\int_{-\pi}^{\pi} W_{\gamma}^{2}(\xi) d \xi=(1 / 2 \pi) \bar{W}(\gamma)$, so we have the variance of the estimator

$$
\begin{aligned}
& \operatorname{Var}\left[\widehat{G}_{\text {NEFOP }}\left(e^{i \omega_{0}}\right)\right] \\
& =\frac{(1 / N) \bar{W}(\gamma) \Phi_{v}\left(\omega_{0}\right)}{\Phi_{u}^{2}\left(\omega_{0}\right)}+o\left(\frac{\bar{W}(\gamma)}{N}\right) \\
& \approx \frac{(1 / N) \bar{W}(\gamma) \Phi_{v}\left(\omega_{0}\right)}{\substack{\gamma \rightarrow \infty, \gamma / N \rightarrow 0 \\
N \rightarrow \infty}}
\end{aligned}
$$

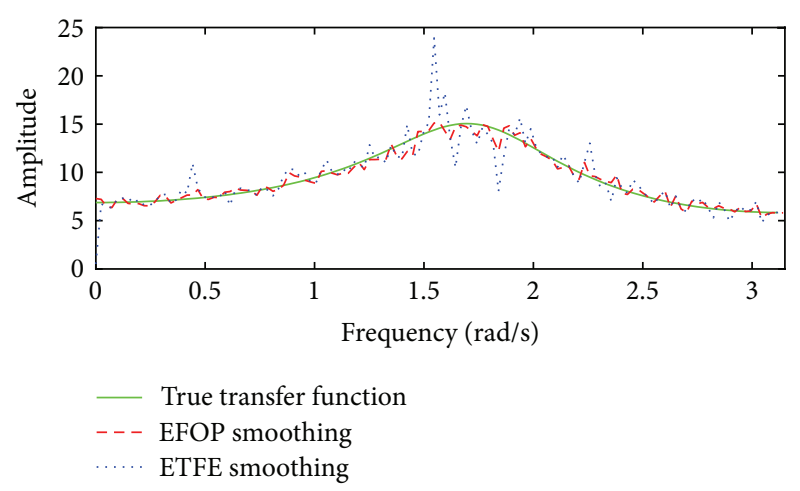

(a)

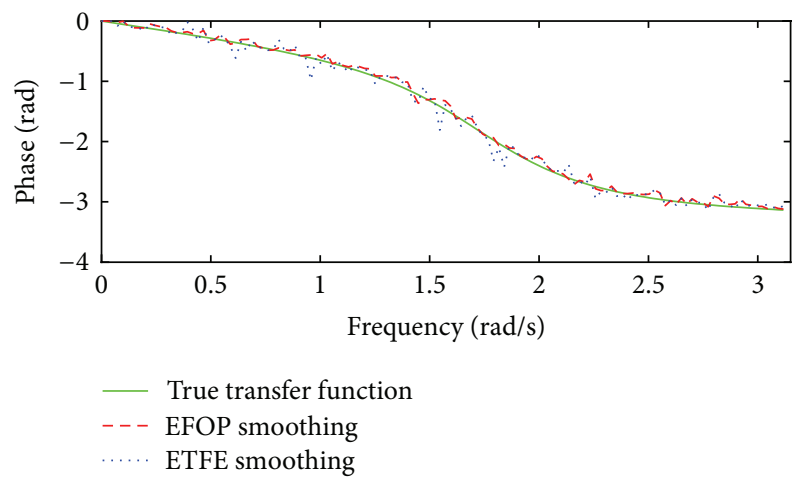

(b)

FIgURE 2: Results of EFOP smoothing and ETFE smoothing for system in (43) under $\sigma_{e}^{2}=0.5$. Error EFOP $^{2}=10.21$; Error $_{\mathrm{ETFE}}=$ 23.35 .

\section{Simulations}

In this section, we use the target system

$$
\begin{aligned}
y(t) & -0.2 y(t-1)+0.4 y(t-2) \\
& =9 u(t-1)+2 u(t-2)+e(t)
\end{aligned}
$$

$\{u(t)\}$ is a white Pseudo-Random Binary Sequence (PRBS) [12] noise with unit variance $\sigma_{u}^{2}=1$. And $e(t)$ is a gaussian white noise with variance $\sigma_{e}^{2}$.

We compared EFOP smoothing with ETFE smoothing and cross-spectral method. In all simulations, a Hamming window of length 2 is used for all methods:

$$
w(n)=0.54-0.46 \cos \left(2 \pi \frac{n}{N}\right) .
$$

And the estimation errors are calculated by the following equation:

$$
\text { error }=\left(\sum_{k=1}^{N}\left|\widehat{G}(k)-G_{0}(k)\right|^{2}\right)^{1 / 2}
$$

where $G_{0}$ is the sample sequence of true transfer function with $N=128$. And $\widehat{G}$ is the estimation result with same length. 
TABLE 1: Estimation error for 3 methods under different noise variances.

\begin{tabular}{lccccccc}
\hline Noise variance & 0.1 & 0.4 & 0.7 & 1.0 & 1.3 & 1.6 & 1.9 \\
\hline Estimate error of ETFE smoothing & 23.34 & 25.62 & 27.14 & 32.86 & 38.27 & 41.31 & 66.48 \\
Estimate error of cross-spectral method & 12.23 & 13.43 & 16.83 & 17.32 & 21.56 & 25.87 & 29.89 \\
Estimate error of EFOP smoothing & 9.88 & 10.68 & 13.14 & 13.96 & 16.67 & 20.86 & 24.79 \\
\hline
\end{tabular}

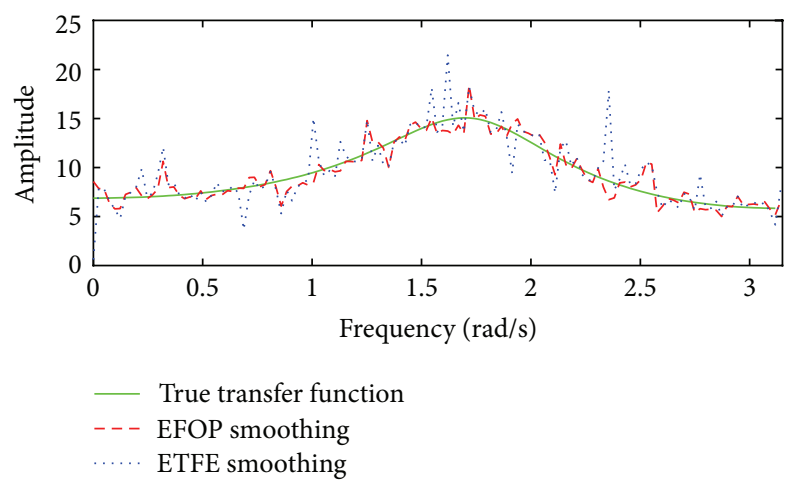

(a)

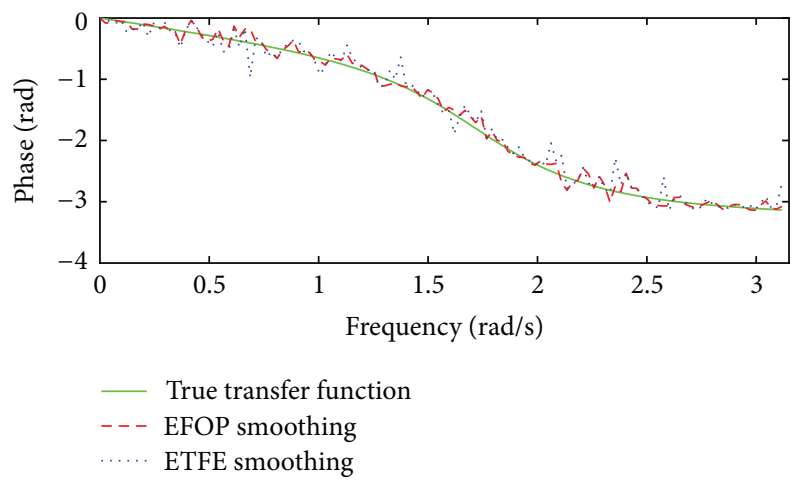

(b)

FIgURE 3: Results of EFOP smoothing and ETFE smoothing for system in (43) under $\sigma_{e}^{2}=1.5$. Error EFOP $=29.07$; Error $_{\mathrm{ETFE}}=$ 42.80 .

In Figures 2 to 5 we compared three methods for frequency domain transfer function estimation. In each figure, the top picture shows the amplitudes of the true and estimated frequency domain transfer function and the bottom picture shows the phase of them. Figures 2 and 3 are comparison between EFOP smoothing and ETFE smoothing under noise variance of 0.5 and 1.5. The estimation error for EFOP smoothing and ETFE smoothing in Figure 2 are 10.21 and 23.35, respectively. And the estimation error for EFOP smoothing and ETFE smoothing in Figure 3 are 29.07 and 42.80, respectively. Figures 4 and 5 are comparison between EFOP smoothing and cross-spectral method under noise variance of 0.5 and 1.5. The estimation error for EFOP smoothing and cross-spectral method in Figure 4 are 11.29 and 13.95, respectively. And the estimation error for EFOP smoothing and Cross Spectral Method in Figure 5 is 17.60 and 20.30 , respectively.

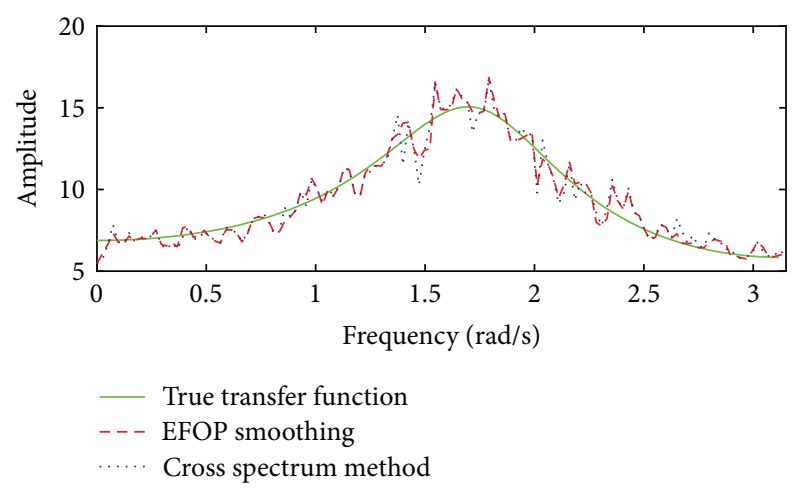

(a)

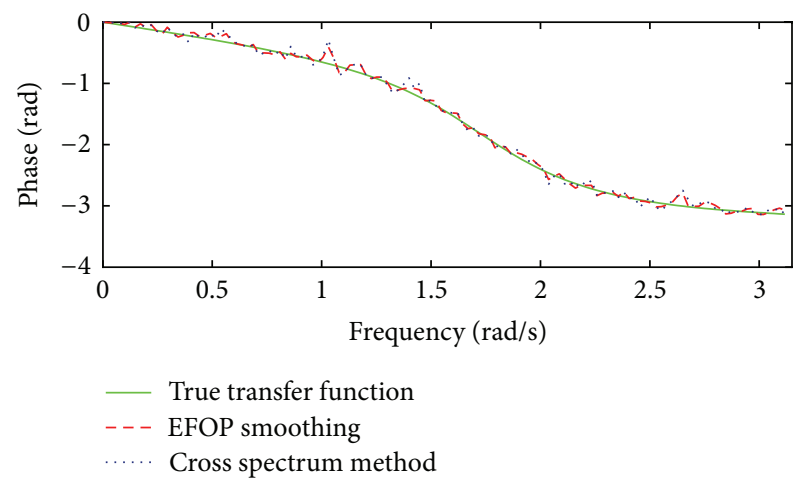

(b)

FIGURE 4: Results of EFOP smoothing and cross-spectral method for system in (43) under $\sigma_{e}^{2}=0.5$. Error $_{\mathrm{EFOP}}=$ 11.29; Error $_{\text {CrossSpectral }}=13.95$.

In all simulations, the variance of input signals are 1 . And all results are obtained by smoothing with a same hamming window of length 2 . It can be seen from the results that EFOP method gives better estimation of the transfer function than ETFE and cross-spectral method. Figure 6 is obtained by numerical simulations. The error values in Figure 6 are also represented in Table 1 . The estimation error increases with noise signal ratio and EFOP smoothing gets estimations of transfer function with lowest error values.

\section{Conclusion}

In this paper, the convergence of transfer function estimation based on integrity form of the GPE criterion with frequency domain window function is analyzed. This smoothing technic gives better results on transfer functions of linear models than traditional ETFE method, especially for systems disturbed by large noises. The consistent property and convergence rate of 


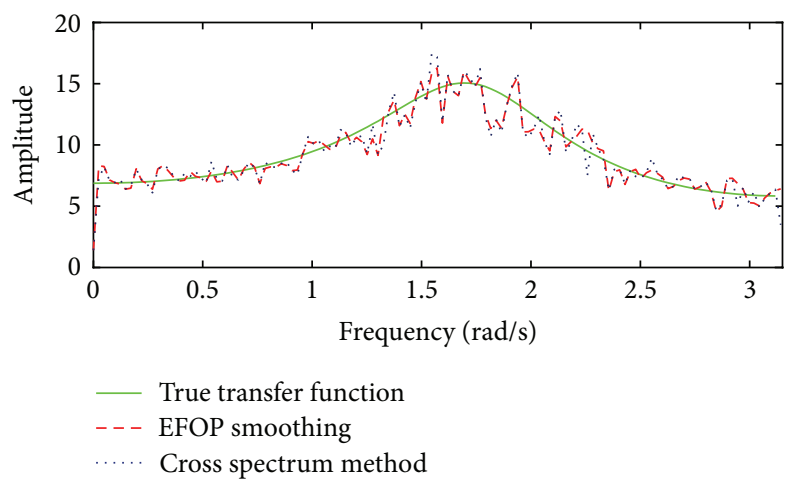

(a)

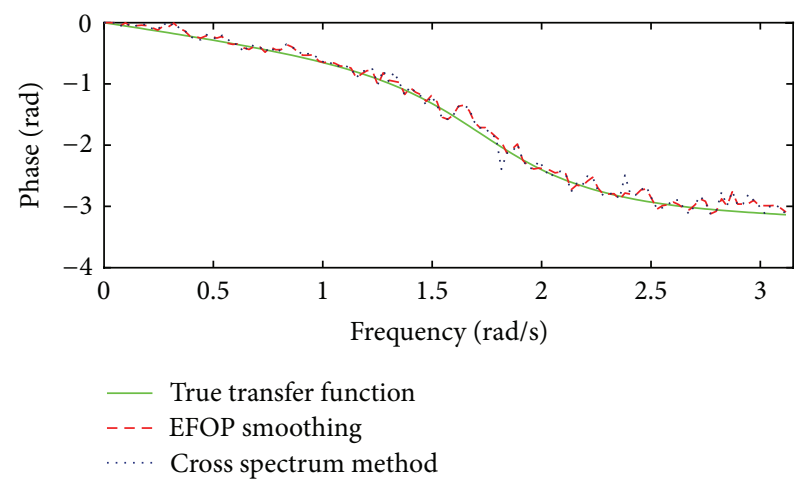

(b)

FIGURE 5: Results of EFOP smoothing and cross-spectral method for system in (43) under $\sigma_{e}^{2}=1.5$ Error $_{\text {EFOP }}=17.60$; Error ${ }_{\text {CrossSpectral }}=$ 20.30 .

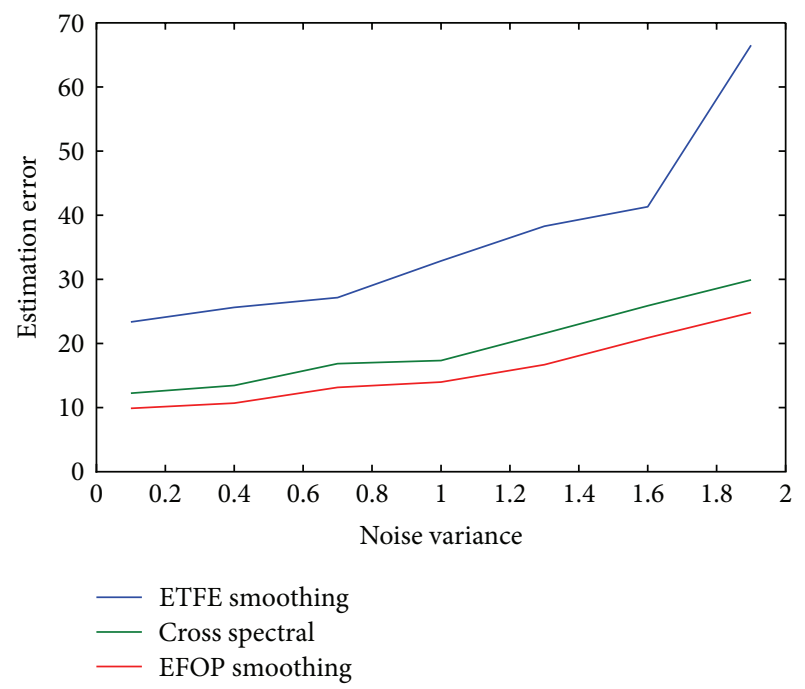

FIGURE 6: Estimate error of EFOP smoothing, ETFE smoothing, and cross-spectral method for system in (43) under $\sigma_{e}^{2}$ from 0.1 to 1.9 .

this method are obtained. Simulations for identification of frequency domain transfer functions are given to illustrate the advantage of this technic derived.

\section{Acknowledgment}

This work is supported by the Funds 863 Project 2012AA040906, NSFC61171121, NSFC60973049, and the Science Foundation of Chinese Ministry of EducationChina Mobile 2012.

\section{References}

[1] L. Ljung, System Identification, Wiley Online Library, 1999.

[2] C. Bingham, M. Godfrey, and J. Tukey, "Modern techniques of power spectrum estimation," IEEE Transactions on Audio and Electroacoustics, vol. 15, no. 2, pp. 56-66, 1967.
[3] P. C. W. Sommen, P. J. van Gerwen, H. J. Kotmans, and A. J. E. M. Janssen, "Convergence analysis of a frequencydomain adaptive filter with exponential power averaging and generalized window function," IEEE Transactions on Circuits and Systems, vol. 34, no. 7, pp. 788-798, 1987.

[4] R. Pintelon and J. Schoukens, System Identification: A Frequency Domain Approach, Wiley-IEEE Press, 2004.

[5] A. Stenman, F. Gustafsson, D. E. Rivera, L. Ljung, and T. McKelvey, "On adaptive smoothing of empirical transfer function estimates," Control Engineering Practice, vol. 8, no. 11, pp. 1309$1315,2000$.

[6] H. Hjalmarsson and B. Ninness, "Least-squares estimation of a class of frequency functions: a finite sample variance expression," Automatica, vol. 42, no. 4, pp. 589-600, 2006.

[7] J. C. Agüero, J. I. Yuz, G. C. Goodwin, and R. A. Delgado, "On the equivalence of time and frequency domain maximum likelihood estimation," Automatica, vol. 46, no. 2, pp. 260-270, 2010.

[8] K. Lo and W. H. Kwon, "A new identification approach for FIR models," IEEE Transactions on Circuits and Systems II, vol. 49, no. 6, pp. 439-446, 2002.

[9] K. Lo and H. Kimura, "Recursive estimation methods for discrete systems," IEEE Transactions on Automatic Control, vol. 48, no. 11, pp. 2019-2023, 2003.

[10] J. C. Agüero, W. Tang, J. I. Yuz, R. Delgado, and G. C. Goodwin, "Dual time-frequency domain system identification," Automatica, vol. 48, no. 12, pp. 3031-3041, 2012.

[11] K. Lo, H. Kimura, W. H. Kwon, and X. Yang, "Empirical frequency-domain optimal parameter estimate for black-box processes,' IEEE Transactions on Circuits and Systems I, vol. 53, no. 2, pp. 419-430, 2006.

[12] H. J. Vermeulen, J. M. Strauss, and V. Shikoana, "Online estimation of synchronous generator parameters using PRBS perturbations," IEEE Transactions on Power Systems, vol. 17, no. 3, pp. 694-700, 2002. 


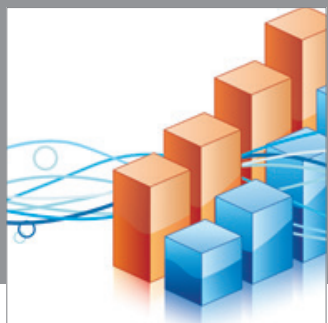

Advances in

Operations Research

mansans

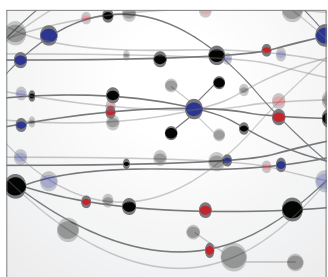

The Scientific World Journal
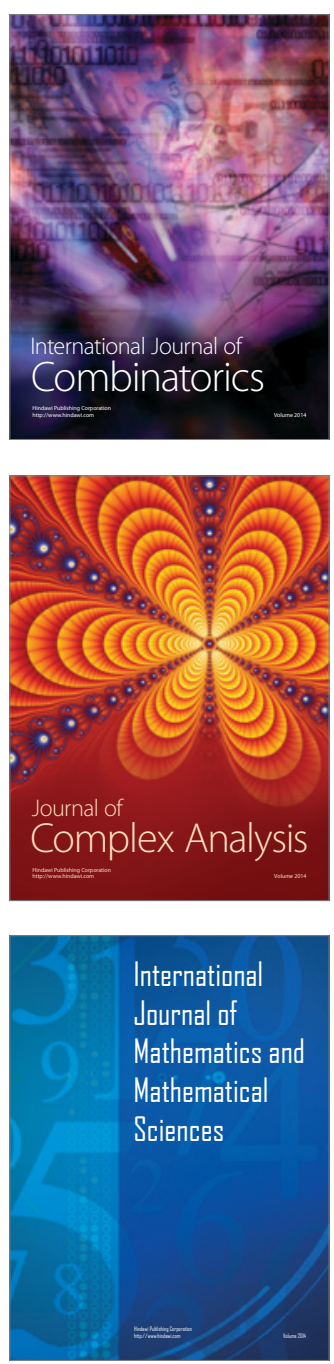
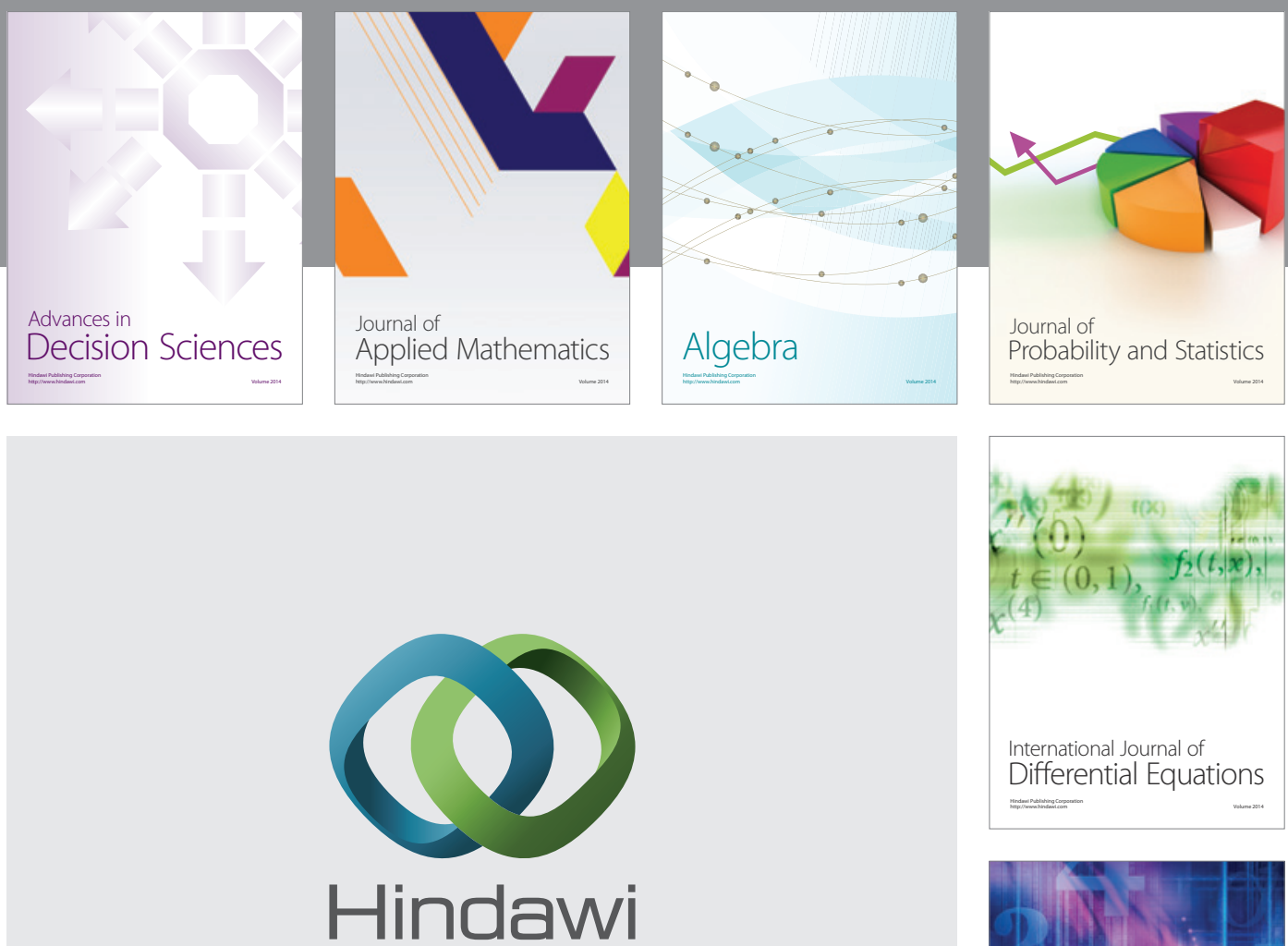

Submit your manuscripts at http://www.hindawi.com
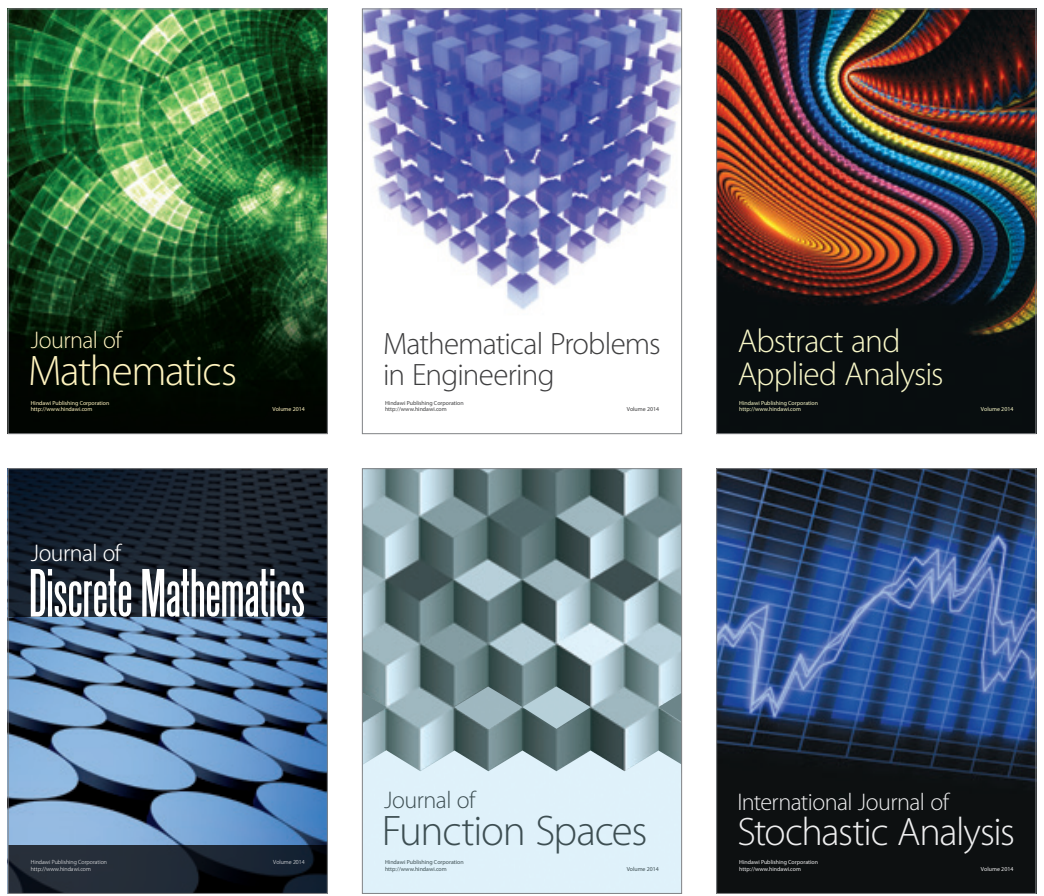

Journal of

Function Spaces

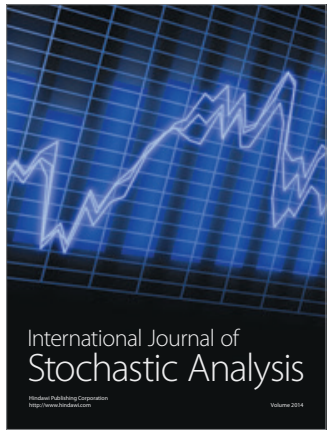

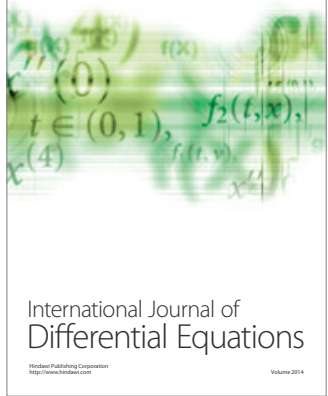
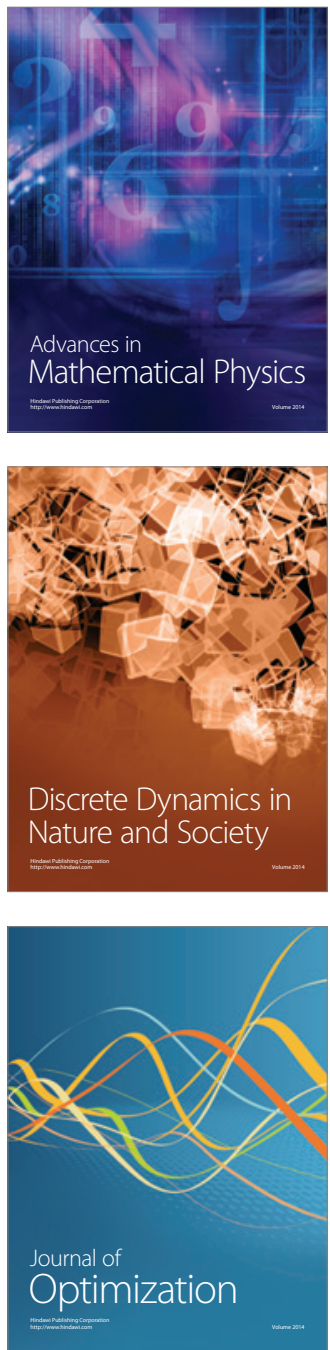